\title{
OBSERVABILITY CRITERIA AND ESTIMATOR DESIGN FOR STOCHASTIC LINEAR HYBRID SYSTEMS
}

\author{
Inseok Hwang, Hamsa Balakrishnan, and Claire Tomlin \\ Hybrid Systems Laboratory \\ Department of Aeronautics and Astronautics \\ Stanford University, Stanford, CA 94305, U.S.A. \\ ishwang, hamsa, tomlinestanford.edu
}

Keywords: observability, estimation, stochastic linear hybrid systems

\begin{abstract}
A stochastic linear hybrid system is said to be observable if the hybrid state of the system can be uniquely determined from its output. In this paper, we derive conditions for the observability of stochastic linear hybrid systems by exploiting the information obtained from system noise characteristics. Having established the necessary criteria for observability, we study the effect of these conditions on estimator design, and also find bounds on the switching times of the system to achieve guaranteed estimator performance. We then apply these results to the estimation of a two-mode aircraft trajectory.
\end{abstract}

\section{Introduction}

The tracking of aircraft trajectories is a problem that has been approached with some success using hybrid system models [1]. Related problems of interest to us are the ability to estimate the hybrid states of such systems from their outputs, and also the design of estimators for such systems. The problem of observability, namely, the ability to estimate or reconstruct the actual state of a system given its output, has been studied extensively, both for continuous [2] and discrete [3, 4] systems. More recently, several researchers have approached the problem of observability of hybrid systems. A practical problem that has received increased attention is the extension of these concepts to stochastic hybrid systems. In this paper, we address the issue of observability of a class of stochastic hybrid systems - systems where the continuous dynamics are affected by white Gaussian noise. Alessandri and Coletta [5] proposed a Luenberger observer design methodology, while Balluchi et al. [6] developed a method of combining location observers for discrete state estimation with Luenberger observers for continuous state estimation for deterministic linear systems. Bemporad et al. [7] defined the concept of incremental observability of continuous-time linear hybrid systems. Recently, Vidal et al. [8] derived observability conditions for linear hybrid systems with continuous-time continuous-state dynamics, given in the form of rank conditions similar to those for continuoustime linear system observability. For stochastic systems, the definition of observability in its classical form, as proposed by Kalman for systems with no noise, fails; we therefore need to find a meaningful interpretation of observability for systems with random noise. Baram and Kailath [9] proposed the con- cept of estimability as a better criterion to gauge stochastic linear systems. While this is one way of approaching the problem, we try to extend the definition of observability to include stochastic hybrid systems.

An important class of problems associated with applications in multi-target tracking [1] and speech recognition [10] pertains to the estimation of discrete-time Markov jump linear systems. Cost and do Val [11] analyzed such systems with finite Markov states and deterministic continuous dynamics. Vidal et al. [12] derived observability conditions for jump linear systems based on rank tests similar to those of deterministic linear hybrid systems. The first part of this study is motivated by the results of Vidal et al. [12]. They proposed the notion of indistinguishability, and derived elegant rank tests for the observability of stochastic jump linear systems, in the mean (or deterministic) sense. Since in the design of estimators for aircraft tracking we have knowledge of not just the system dynamics, but also the noise covariances, we try to exploit this additional knowledge to improve our ability to differentiate between state trajectories. Since the output sequences of stochastic systems might be different from the same initial condition, we extend the notion of indistinguishability [12] for such systems, and based on our definition, we derive conditions for the observability of discrete-time stochastic linear hybrid systems. The latter part of this paper applies the approach of Balluchi et al. [6], so far used in the design of hybrid observers for deterministic hybrid systems with continuous-time state evolution, to discrete-time stochastic hybrid systems and estimator design.

This paper is organized as follows: Section 2 presents the observability conditions of discrete-time stochastic jump linear systems. In Section 3, we obtain conditions on the system parameters that would guarantee the exponential convergence of hybrid estimators. Examples and conclusions are presented in Sections 4 and 5 respectively. Proofs of theorems are not included due to limited space; they are available upon request.

\section{Observability of discrete-time stochastic lin- ear hybrid systems}

In this section, inspired by Vidal et al. [12], we extend the concepts of indistinguishability, observability of the hybrid initial state, and discrete transition times as defined in [12] and derive more general observability conditions for discrete-time stochastic linear hybrid systems using the knowledge of noise covariances. We consider a discrete-time stochastic linear hy- 
brid system

$$
H:\left\{\begin{array}{c}
x_{k+1}=A\left(q_{k}\right) x_{k}+w_{k}\left(q_{k}\right) \\
y_{k}=C\left(q_{k}\right) x_{k}+v_{k}\left(q_{k}\right) \quad, k \in\{0,1, \cdots\} \\
q_{k+1}=\delta\left(q_{k}, \gamma_{k}\right)
\end{array}\right.
$$

where $k$ is a non-negative integer $(k \in \mathbb{N}) ; x_{k} \in \mathbb{R}^{n}$ and $y_{k} \in$ $\mathbb{R}^{p}$ are the continuous state and output variables respectively; $q_{k} \in\{1,2, \cdots, N\}$ is the discrete state, $\gamma_{k} \in\left\{\gamma^{1}, \cdots, \gamma^{m}\right\}$ is a discrete control input, and $\delta(\cdot, \cdot)$ is a deterministic discrete transition relation which governs the discrete state evolution. We assume the event time at which a discrete transition occurs is unknown. The system parameters $A\left(q_{k}\right) \in \mathbb{R}^{n \times n}$ and $C\left(q_{k}\right) \in \mathbb{R}^{p \times n}$ for $q_{k} \in\{1,2, \cdots, N\}$ are real matrices. We assume that the initial state $x_{k_{0}}$ is an unknown, zero-mean white Gaussian random variable with covariance $E\left[x_{k_{0}} x_{k_{0}}^{T}\right]=$ $\Pi_{0}$ and that the process noise $w_{k}\left(q_{k}\right)$ and the measurement noise $v_{k}\left(q_{k}\right)$ are uncorrelated, zero-mean white Gaussian sequences with the covariance matrices $E\left[w_{k}\left(q_{k}\right) w_{k}\left(q_{k}\right)^{T}\right]=$ $\rho\left(q_{k}\right) I$ and $E\left[v_{k}\left(q_{k}\right) v_{k}\left(q_{k}\right)^{T}\right]=\sigma\left(q_{k}\right) I$ respectively. These random sequences are assumed to be uncorrelated with the initial state, i.e., $E\left[x_{k_{0}} w_{k}\left(q_{k}\right)^{T}\right]=E\left[x_{k_{0}} v_{k}\left(q_{k}\right)^{T}\right]=0 . I$ denotes the identity matrix. Since the state evolution of a hybrid system has continuous trajectories as well as discrete jumps, we define a hybrid time trajectory:

Definition 1 (Hybrid time trajectory) A hybrid time trajectory is a sequence of intervals $\left[k_{0}, k_{1}-1\right]\left[k_{1}, k_{2}-1\right] \cdots\left[k_{i}, k_{i+1}-\right.$ $1] \cdots$ where $k_{i}(i \geq 1)$ is the time at which the $i$-th discrete state transition occurs.

Before deriving the observability conditions, we review the definition of observability for discrete-time stochastic linear hybrid systems [12]:

Definition 2 (Observability of discrete-time stochastic linear hybrid systems) A discrete-time linear hybrid system $H$ is observable on $\left[k_{0}, k_{0}+K\right]$ if the hybrid state $\left(q_{k}, x_{k}\right)$ for $k \in\left[k_{0}, k_{0}+K\right]$ is uniquely determined (in the case of $x_{k}$ as a unique probability distribution), from the output sequence $\mathcal{Y}_{K}=\left[y_{k_{0}}^{T} \cdots y_{k_{0}+K}^{T}\right]^{T}$, where $K \in \mathbb{N}$.

Vidal et al. [12] developed rank tests for the observability of stochastic jump linear systems of the form described by $H$ (Eq.(1)) using the notion of indistinguishability. Since we know the noise covariances as well as the system dynamics for a stochastic system, we use this additional knowledge to obtain a more general condition. Since the output sequences of stochastic systems could be different from the same initial condition, we extend the notion of indistinguishability [12] as follows:

Definition 3 (Indistinguishability of discrete-time stochastic linear hybrid systems) A discrete-time linear hybrid system $H$ is indistinguishable on $\left[k_{0}, k_{0}+K\right]$ if there exist output sequences $\mathcal{Y}_{K}$ and $\mathcal{Y}_{K}^{\prime}$ on $k \in\left[k_{0}, k_{0}+K\right]$ starting from any two different hybrid states $\left(q_{k_{0}}, x_{k_{0}}\right)$ and $\left(q_{k_{0}}^{\prime}, x_{k_{0}}^{\prime}\right)$, which are identically distributed.

\subsection{Observability of the hybrid initial state}

In this section, using a procedure similar to that in [12], we derive the conditions under which the hybrid initial state $\left(q_{k_{0}}, x_{k_{0}}\right)$ can be uniquely determined from the output sequence $\left\{y_{k}\right\}$ on $\left[k_{0}, k_{1}-1\right]\left(k_{1}-1 \leq k_{0}+K\right)$, i.e., before the first discrete transition occurs. We define $\kappa_{i}:=k_{i+1}-k_{i}$ $(i \geq 0)$ as the sojourn time, which denotes how long the system stays in a discrete state after the $i$-th discrete transition. Based on Definition 2 and Definition 3, we get the following lemma:

Lemma 1 The hybrid initial state of a discrete-time linear hybrid system $H$ is observable if and only if it is distinguishable.

In order to check if the hybrid initial state is distinguishable, we need to compare the distributions of the output sequences. In the case of a linear system with Gaussian noise, this task is greatly simplified, since the distributions are uniquely determined by their means and covariances. A difference in the means reduces to observability in the deterministic case, and we can test this in several ways; in particular [12] presents elegant rank conditions for this purpose, henceforth referred to as the Vidal-Chiuso-Soatto (VCS) conditions.

Lemma 2 The hybrid initial state of a discrete-time linear stochastic hybrid system is observable if the equivalent deterministic system is observable, i.e, the VCS conditions are satisfied.

If the VCS conditions are not satisfied, we have to compute the covariances (higher moments) of the output sequence $\mathcal{Y}_{\kappa_{0}}$ on $\left[k_{0}, k_{1}-1\right]$. The output sequence starting from the hybrid initial state $\left(q_{k_{0}}, x_{k_{0}}\right)$ on $\left[k_{0}, k_{1}-1\right]$ is

$$
\begin{aligned}
& \mathcal{Y}_{\kappa_{0}}\left(q_{k_{0}}\right)=\mathcal{O}_{\kappa_{0}}\left(q_{k_{0}}\right) x_{k_{0}}+\mathcal{T}_{\kappa_{0}}\left(q_{k_{0}}\right) W_{\kappa_{0}}\left(q_{k_{0}}\right)+V_{\kappa_{0}}\left(q_{k_{0}}\right) \\
& \text { where } \mathcal{O}_{\kappa_{0}}\left(q_{k_{0}}\right)=\left[C\left(q_{k_{0}}\right)^{T}\left(C\left(q_{k_{0}}\right) A\left(q_{k_{0}}\right)\right)^{T} \ldots\right. \\
& \left.\cdots\left(\left(C\left(q_{k_{0}}\right) A\left(q_{k_{0}}\right)\right)^{k_{1}-1}\right)^{T}\right]^{T} \\
& \mathcal{T}_{\kappa_{0}}\left(q_{k_{0}}\right)=\left[\begin{array}{ccc}
0 & \cdots & 0 \\
C\left(q_{k_{0}}\right) & \cdots & 0 \\
C\left(q_{k_{0}}\right) A\left(q_{k_{0}}\right) & \cdots & 0 \\
\vdots & & \\
C\left(q_{k_{0}}\right) A\left(q_{k_{0}}\right)^{k_{1}-k_{0}-2} & \cdots & 0
\end{array}\right] \\
& W_{\kappa_{0}}\left(q_{k_{0}}\right)=\left[w_{k_{0}}\left(q_{k_{0}}\right)^{T} w_{k_{0}+1}\left(q_{k_{0}}\right)^{T} \cdots w_{k_{1}-1}\left(q_{k_{0}}\right)^{T}\right]^{T} \\
& V_{\kappa_{0}}\left(q_{k_{0}}\right)=\left[v_{k_{0}}\left(q_{k_{0}}\right)^{T} v_{k_{0}+1}\left(q_{k_{0}}\right)^{T} \cdots v_{k_{1}-1}\left(q_{k_{0}}\right)^{T}\right]^{T}
\end{aligned}
$$
the linear system in Eq.(1) [12] and $\mathcal{T}_{\kappa_{0}}\left(q_{k_{0}}\right)$ is a Toeplitz matrix.

If $\operatorname{rank}\left[\mathcal{O}_{\kappa_{0}}\left(q_{k_{0}}\right)\right]=n$, i.e., the linear system $\left(A\left(q_{k_{0}}\right), C\left(q_{k_{0}}\right)\right)$ is observable and $\kappa_{0} \geq n$, then a leastsquares solution (which we denote by $\hat{x}_{k_{0}}\left(q_{k_{0}}\right)$ ) to Eq.(2) can be determined uniquely.

$$
\begin{aligned}
\hat{x}_{k_{0}}\left(q_{k_{0}}\right)= & \mathcal{O}_{\kappa_{0}}^{\dagger}\left(q_{k_{0}}\right) \mathcal{Y}_{\kappa_{0}}\left(q_{k_{0}}\right) \\
= & x_{k_{0}}+\mathcal{O}_{\kappa_{0}}^{\dagger}\left(q_{k_{0}}\right) \mathcal{T}_{\kappa_{0}}\left(q_{k_{0}}\right) W_{\kappa_{0}}\left(q_{k_{0}}\right) \\
& +\mathcal{O}_{\kappa_{0}}^{\dagger}\left(q_{k_{0}}\right) V_{\kappa_{0}}\left(q_{k_{0}}\right)
\end{aligned}
$$

where $\mathcal{O}_{\kappa_{0}}^{\dagger}\left(q_{k_{0}}\right)=\left(\mathcal{O}_{\kappa_{0}}^{T}\left(q_{k_{0}}\right) \mathcal{O}_{\kappa_{0}}\left(q_{k_{0}}\right)\right)^{-1} \mathcal{O}_{\kappa_{0}}^{T}\left(q_{k_{0}}\right)$. The last two terms on the right hand side of Eq.(3) represent the estimation error due to the process noise and the measurement noise. 
Similarly, the output sequence from another hybrid initial state $\left(q_{k_{0}}^{\prime}, x_{k_{0}}^{\prime}\right)$ over $\left[k_{0}, k_{1}-1\right]$ is

$$
\mathcal{Y}_{\kappa_{0}}\left(q_{k_{0}}^{\prime}\right)=\mathcal{O}_{\kappa_{0}}\left(q_{k_{0}}^{\prime}\right) x_{k_{0}}^{\prime}+\mathcal{T}_{\kappa_{0}}\left(q_{k_{0}}^{\prime}\right) W_{\kappa_{0}}\left(q_{k_{0}}^{\prime}\right)+V_{\kappa_{0}}\left(q_{k_{0}}^{\prime}\right)
$$

From Lemma 1, in order that the hybrid initial state of a discrete-time stochastic linear hybrid system be observable, it should be distinguishable, i.e., if Lemma $\mathbf{2}$ is not satisfied, the covariances of $\mathcal{Y}_{\kappa_{0}}\left(q_{k_{0}}\right)$ and $\mathcal{Y}_{\kappa_{0}}\left(q_{k_{0}}^{\prime}\right)$ satisfy:

$$
E\left[\mathcal{Y}_{\kappa_{0}}\left(q_{k_{0}}\right) \mathcal{Y}_{\kappa_{0}}\left(q_{k_{0}}\right)^{T}\right] \neq E\left[\mathcal{Y}_{\kappa_{0}}\left(q_{k_{0}}^{\prime}\right) \mathcal{Y}_{\kappa_{0}}\left(q_{k_{0}}^{\prime}\right)^{T}\right]
$$

where

$$
\begin{aligned}
& E\left[\mathcal{Y}_{\kappa_{0}}\left(q_{k_{0}}\right) \mathcal{Y}_{\kappa_{0}}\left(q_{k_{0}}\right)^{T}\right]=\mathcal{O}_{\kappa_{0}}\left(q_{k_{0}}\right) \Pi_{0} \mathcal{O}_{\kappa_{0}}\left(q_{k_{0}}\right)^{T} \\
+\quad & \rho\left(q_{k_{0}}\right) \mathcal{T}_{\kappa_{0}}\left(q_{k_{0}}\right) \mathcal{T}_{\kappa_{0}}^{T}\left(q_{k_{0}}\right)+\sigma\left(q_{k_{0}}\right) I \\
& E\left[\mathcal{Y}_{\kappa_{0}}\left(q_{k_{0}}^{\prime}\right) \mathcal{Y}_{\kappa_{0}}\left(q_{k_{0}}^{\prime}\right)^{T}\right]=\mathcal{O}_{\kappa_{0}}\left(q_{k_{0}}^{\prime}\right) \Pi_{0} \mathcal{O}_{\kappa_{0}}\left(q_{k_{0}}^{\prime}\right)^{T} \\
+ & \rho\left(q_{k_{0}}^{\prime}\right) \mathcal{T}_{\kappa_{0}}\left(q_{k_{0}}^{\prime}\right) \mathcal{T}_{\kappa_{0}}^{T}\left(q_{k_{0}}^{\prime}\right)+\sigma\left(q_{k_{0}}^{\prime}\right) I
\end{aligned}
$$

Then, the discrete initial state can be uniquely determined from the covariance of the output sequence and the continuous initial state can also be uniquely determined using Eq.(3). In order to reduce the required $\kappa_{0}$ for observability (the sojourn time in the discrete state $q_{k_{0}}$ required for observability of the hybrid initial state), we define $\tau$ as the minimum integer which satisfies $\operatorname{rank}\left[\mathcal{O}_{\tau}\left(q_{k}\right)\right]=n\left(\forall q_{k} \in\{1,2, \cdots, N\}\right)$, and $\bar{\tau}=\max \tau$ (similar to the joint observability index used in [12]). Then, we have the following condition for the observability of the hybrid initial state:

Lemma 3 (Observability of the hybrid initial state) If $\left(A\left(q_{k}\right), C\left(q_{k}\right)\right)$ are observable for each $q_{k} \in\{1, \cdots, N\}$ and $\kappa_{0} \geq \bar{\tau}$, the hybrid initial state $\left(q_{k_{0}}, x_{k_{0}}\right)$ is observable if and only if either the hybrid initial state of the deterministic system is observable, or

$\mathcal{O}_{\bar{\tau}}\left(q_{k_{0}}\right) \Pi_{0} \mathcal{O}_{\bar{\tau}}\left(q_{k_{0}}\right)^{T}+\rho\left(q_{k_{0}}\right) \mathcal{T}_{\bar{\tau}}\left(q_{k_{0}}\right) \mathcal{T}_{\bar{\tau}}^{T}\left(q_{k_{0}}\right)+\sigma\left(q_{k_{0}}\right) I$ $\neq \mathcal{O}_{\bar{\tau}}\left(q_{k_{0}}^{\prime}\right) \Pi_{0} \mathcal{O}_{\bar{\tau}}\left(q_{k_{0}}^{\prime}\right)^{T}+\rho\left(q_{k_{0}}^{\prime}\right) \mathcal{I}_{\bar{\tau}}\left(q_{k_{0}}^{\prime}\right) \mathcal{T}_{\bar{\tau}}^{T}\left(q_{k_{0}}^{\prime}\right)+\sigma\left(q_{k_{0}}^{\prime}\right) I$ for all $q_{k_{0}} \neq q_{k_{0}}^{\prime} \in\{1, \cdots, N\}$.

We show through the following simple example how a noise free unobservable discrete-time linear hybrid system may be rendered observable, if each discrete state is endowed with different measurement noise covariances.

Example: Consider a discrete-time linear hybrid system with two discrete states

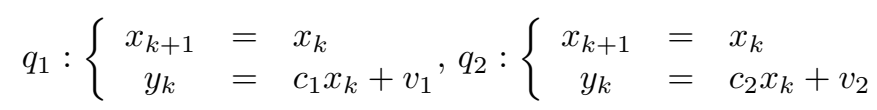

where $c_{1} \neq 0, c_{2} \neq 0$, and $c_{1} \neq c_{2}$. The covariance of the initial state is $E\left[x_{0} x_{0}^{T}\right]=\pi_{0} \in \mathbb{R}^{+} . \quad v_{1}$ and $v_{2}$ are uncorrelated, zero-mean white Gaussian sequences with covariances $E\left[v_{1} v_{1}^{T}\right]=\sigma_{1} \neq 0$ and $E\left[v_{2} v_{2}^{T}\right]=\sigma_{2} \neq 0$ respectively. If $v_{1}=v_{2}=0$, the hybrid system is unobservable because two different hybrid initial states $\left(q_{1}, x_{0}\right)$ and $\left(q_{2}, \frac{c_{1}}{c_{2}} x_{0}\right)$ generate the same output sequences [8]. However, if $v_{1}$ and $v_{2}$ are not identically zero and have different covariances, then we can uniquely determine the hybrid initial state. If we consider the case in which the actual hybrid initial state is $\left(q_{1}, x_{0}\right)$, the output and its covariance are $y=c_{1} x_{0}+v_{1}, \quad E\left[y y^{T}\right]=\pi_{0} c_{1} c_{1}^{T}+\sigma_{1}$.

Next, if the actual hybrid initial state is $\left(q_{2}, \frac{c_{1}}{c_{2}} x_{0}\right)$, the output and its covariance are $y=c_{2}\left(\frac{c_{1}}{c_{2}} x_{0}\right)+v_{2}, \quad E\left[y y^{T}\right]=\pi_{0} c_{1} c_{1}^{T}+\sigma_{2}$.

Since $\sigma_{1} \neq \sigma_{2}$, we can determine the discrete initial state uniquely. For instance, if the output comes from $q_{1}$, then the estimate of the initial state is $\hat{x}_{0}=x_{0}+\frac{v_{1}}{c_{1}}$.

\subsection{Observability of the discrete transition times}

Lemma 3 gives the condition for the hybrid initial state to be observable, over a time interval up to, but not including the first transition. In this section, we focus without loss of generality on deriving the conditions under which the first discrete transition time $k_{1}$ can be uniquely determined from the output sequence $\mathcal{Y}_{K}$ on $\left[k_{0}, k_{0}+K\right]$; similarly, the times of the ensuing transitions $k_{i}(i \in\{2, \ldots\})$ can be computed [12].

We define observability of the first discrete transition time as follows:

Definition 4 (Observability of the first discrete transition time) The first discrete transition time of a discrete-time linear hybrid system $H$ is observable on $\left[k_{0}, k_{0}+K\right]$ if it can be determined uniquely from the output sequence $\mathcal{Y}_{K}=\left[y_{k_{0}}^{T} \cdots y_{k_{0}+K}^{T}\right]^{T}$.

If there is a discrete transition at time $k_{1} \in\left[k_{0}, k_{0}+K\right]$, the output at time $k_{1}$ and its covariance are

$$
\begin{aligned}
y_{k_{1}}= & C\left(q_{k_{1}}\right) A\left(q_{k_{0}}\right)^{k_{1}-k_{0}} x_{k_{0}} \\
& +C\left(q_{k_{1}}\right) \mathcal{F}_{\kappa_{0}}\left(q_{k_{0}}\right) W_{\kappa_{0}}\left(q_{k_{0}}\right)+v_{k_{1}}\left(q_{k_{1}}\right) \\
E\left[y_{k_{1}} y_{k_{1}}^{T}\right]= & C\left(q_{k_{1}}\right) A\left(q_{k_{0}}\right)^{k_{1}-k_{0}} \Pi_{0}\left(A\left(q_{k_{0}}\right)^{k_{1}-k_{0}}\right)^{T} C\left(q_{k_{1}}\right)^{T} \\
& +\rho\left(q_{k_{0}}\right) C\left(q_{k_{1}}\right) \mathcal{F}_{\kappa_{0}}\left(q_{k_{0}}\right) \mathcal{F}_{\kappa_{0}}\left(q_{k_{0}}\right)^{T} C\left(q_{k_{1}}\right)^{T} \\
& +\sigma\left(q_{k_{1}}\right) I
\end{aligned}
$$

where $\mathcal{F}_{\kappa_{0}}\left(q_{k_{0}}\right):=\left[A\left(q_{k_{0}}\right)^{k_{1}-k_{0}-1} A\left(q_{k_{0}}\right)^{k_{1}-k_{0}-2} \ldots I\right]$. If there is no state transition at time $k_{1}$, the output at time $k_{1}$ and its covariance are

$$
\begin{aligned}
y_{k_{1}}= & C\left(q_{k_{0}}\right) A\left(q_{k_{0}}\right)^{k_{1}-k_{0}} x_{k_{0}} \\
& +C\left(q_{k_{0}}\right) \mathcal{F}_{\kappa_{0}}\left(q_{k_{0}}\right) W_{\kappa_{0}}\left(q_{k_{0}}\right)+v_{k_{1}}\left(q_{k_{0}}\right) \\
E\left[y_{k_{1}} y_{k_{1}}^{T}\right]= & C\left(q_{k_{0}}\right) A\left(q_{k_{0}}\right)^{k_{1}-k_{0}} \Pi_{0}\left(A\left(q_{k_{0}}\right)^{k_{1}-k_{0}}\right)^{T} C\left(q_{k_{0}}\right)^{T} \\
& +\rho\left(q_{k_{0}}\right) C\left(q_{k_{0}}\right) \mathcal{F}_{\kappa_{0}}\left(q_{k_{0}}\right) \mathcal{F}_{\kappa_{0}}\left(q_{k_{0}}\right)^{T} C\left(q_{k_{0}}\right)^{T} \\
& +\sigma\left(q_{k_{0}}\right) I
\end{aligned}
$$

In order that the transition at time $k_{1}$ be observable, either means (deterministic observability) or the covariances of $y_{k_{1}}$ 's in Eq.(6) and Eq.(7) should be different. Thus, the observability condition of the first discrete transition time is:

Lemma 4 (Observability of the first discrete transition time) The first discrete transition time is observable if and only if either the first discrete transition time of the equivalent deterministic hybrid system is observable, or

$$
\begin{aligned}
& C\left(q_{k_{1}}\right) A\left(q_{k_{0}}\right)^{k_{1}-k_{0}} \Pi_{0}\left(A\left(q_{k_{0}}\right)^{k_{1}-k_{0}}\right)^{T} C\left(q_{k_{1}}\right)^{T} \\
& +\rho\left(q_{k_{0}}\right) C\left(q_{k_{1}}\right) \mathcal{F}_{\kappa_{0}}\left(q_{k_{0}}\right) \mathcal{F}_{\kappa_{0}}\left(q_{k_{0}}\right)^{T} C\left(q_{k_{1}}\right)^{T}+\sigma\left(q_{k_{1}}\right) I \\
\neq & C\left(q_{k_{0}}\right) A\left(q_{k_{0}}\right)^{k_{1}-k_{0}} \Pi_{0}\left(A\left(q_{k_{0}}\right)^{k_{1}-k_{0}}\right)^{T} C\left(q_{k_{0}}\right)^{T} \\
& +\rho\left(q_{k_{0}}\right) C\left(q_{k_{0}}\right) \mathcal{F}_{\kappa_{0}}\left(q_{k_{0}}\right) \mathcal{F}_{\kappa_{0}}\left(q_{k_{0}}\right)^{T} C\left(q_{k_{0}}\right)^{T}+\sigma\left(q_{k_{0}}\right) I
\end{aligned}
$$

for all $q_{k} \neq q_{k}^{\prime} \in\{1, \cdots, N\}$.

Therefore, from Lemma 2 , Lemma 3 and Lemma 4, the hybrid initial state and the first discrete transition time can be uniquely determined. The remaining state trajectories can be 
determined by repeating the procedure. For $k_{i}(i \geq 1)$, the $\hat{x}_{k_{i}}$ will be given from the initial state estimate [12]. Thus, we have the following observability condition:

Theorem 1 A discrete-time linear stochastic hybrid system $H$ with Gaussian noise is observable if and only if it satisfies either Lemma 2 or Lemma 3 and Lemma 4.

This test needs the operations of multiplication and addition of matrices (which are system parameters and noise covariances): the computation is straightforward with computational complexity depending on data size.

\section{Design of estimators for stochastic hybrid sys- tems}

Having established conditions for the observability of stochastic linear hybrid systems, we would like to design estimators for those observable systems, and also quantify values of system parameters that would guarantee performance (exponential convergence, in our case). We extend the design methods proposed by Balluchi et al. [6] for hybrid systems with continuous-time, continuous state dynamics to encompass discrete-time stochastic hybrid systems.

A hybrid estimator finds estimates $\hat{q}$ and $\hat{x}$ for the current discrete state $q$ and the continuous state $x$ respectively. In this section, we first describe the structure of the hybrid estimator, and then analyze the continuous component of the estimator in detail to obtain bounds on the time between discrete transitions of state which would guarantee exponential convergence of our hybrid estimator. Throughout this paper, all norms, unless specified otherwise, are 2-norms.

Definition 5 (Exponential convergence of a hybrid estimator) Given a hybrid system $H$ with $N$ discrete modes, we say that a hybrid estimator is exponentially convergent if its discrete state estimate $\hat{q}$ exhibits correct identification of the discrete-state transition sequence of the original system after a finite number of steps; and at any given time, the continuous state estimate is unique, with an estimation error mean $\bar{\zeta}=E[\hat{x}-x]$ that converges exponentially to the set $\|\bar{\zeta}\| \leq M_{0}$ with a rate of convergence $\mu$, where $M_{0}$ is the steady-state error bound, and $|\mu|<1$.

\subsection{Structure of the hybrid estimator}

We design the hybrid estimator as a combination of a discrete observer to detect the discrete state switches, and an estimator to estimate the continuous dynamics, as proposed in [6]. In the rest of this paper, we assume that we have a discrete observer that correctly identifies the discrete state, either immediately after a switch takes place, or with a known time delay $\Delta$ after a discrete transition. A discrete observer could be constructed using a bank of $N$ estimators as a residual generator $[1,6]$ - even in this case, we could further increase the probability of correct discrete-state identification by enforcing a decision time delay $\Delta$ on the discrete observer. This would be possible only if the system were observable in the sense of a stochastic hybrid system, as explained earlier.

In this section, we design a least-squares estimator in the form of $N$ Kalman filters for the continuous state estimate. Although the underlying system in [6] is continuous-time and deterministic, the design methodology of [6] adapts well to discrete-time stochastic hybrid systems, as we show here.

\subsection{Discrete-time Kalman filter}

We consider a hybrid system of the form described in Eq.(1). For the sake of simplicity of notation, we replace $A\left(q_{k}\right)$ and $C\left(q_{k}\right)$ with $A_{l}$ and $C_{l}$, where $l \in\{1 \ldots N\}$. We can then write the equations for the least-square estimator of a linear stochastic system as

$$
\hat{x}_{k+1}=\left(A_{l}-K_{l} C_{l}\right) \hat{x}_{k}+K_{P, k, l} y_{k}, k \geq 0
$$

where $l$ is the estimated discrete state, and $K_{P, k, l}$ is the optimal Kalman filter gain for mode $l$, given by $K_{P, k, l}=$ $A_{l} P_{k} C_{l}^{T}\left(R_{l}+C_{l} P_{k} C_{l}^{T}\right)^{-1}$ and $P_{k}$ satisfies the discrete Riccati recursion,

$$
\begin{aligned}
& P_{k+1}=A_{l} P_{k} A_{l}^{T}+Q_{l}-K_{P, k, l}\left(R_{l}+C_{l} P_{k} C_{l}^{T}\right) K_{P, k, l}^{T} \\
& P(0) \triangleq \Pi_{0}
\end{aligned}
$$

The Discrete Algebraic Riccati Equation (DARE) has a stabilizing solution that is unique if and only if $\left\{A_{l}, C_{l}\right\}$ is detectable and $\left\{A_{l}, Q_{l}^{1 / 2}\right\}$ is controllable on the unit circle. Any such solution is positive definite [13]. If these conditions are satisfied for every discrete state $i \in\{1 \ldots N\}$, we can design a bank of $N$ steady-state, exponentially convergent Kalman filters to estimate the continuous state of the system.

We can then show that, for a given discrete state $i$, correctly identified,

$$
\begin{aligned}
\hat{x}_{k+1} & =\left(A_{l}-K_{l} C_{l}\right) \hat{x}_{k}+K_{l} y_{k} \\
\hat{\zeta}_{k+1} & =\left(A_{l}-K_{l} C_{l}\right) \hat{\zeta}_{k}
\end{aligned}
$$

Clearly, $\hat{\zeta}$ is exponentially convergent if

$$
\left(A_{l}-K_{l} C_{l}\right) \text { is stable }
$$

\subsection{Error dynamics}

In this section, we follow the methodology of [6] to determine the evolution of the estimation error across the discrete transition sequence. Let us consider two consequent discrete transitions of $H$, occurring at times $k_{i}$ and $k_{i+1}$. Suppose the transition at time $k_{i+1}$ was from discrete state $m$ to $l$, and was detected at time $k_{i+1}^{\prime}$ such that $k_{i+1}^{\prime}-k_{i+1} \leq \Delta$. Similarly, $k_{i}^{\prime}-k_{i} \leq \Delta$. This is illustrated in Figure 1. We are interested

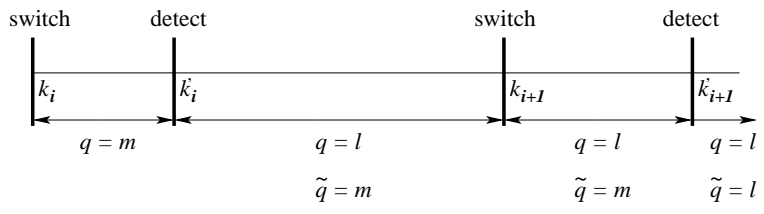

Figure 1: Illustration of the transition sequence

in the region $k \in\left\{k_{i}^{\prime}, k_{i}^{\prime}+1, \ldots, k_{i+1}^{\prime}\right\}$. Since we assume that by time-step $k_{i}^{\prime}$ the discrete state has been identified correctly, for the exponential convergence of the estimation error on $k_{i}^{\prime}$ to $k_{i+1}^{\prime}$, we require that: 
1. The error converges exponentially between $k_{i}^{\prime}$ and $k_{i+1}$;

2. The error divergence between $k_{i+1}$ and $k_{i+1}^{\prime}$ due to wrong discrete state estimation does not upset the exponential convergence of the error on $k_{i}^{\prime}$ to $k_{i+1}^{\prime}$.

Following the methodology of [6], dividing the time interval between $k_{i}^{\prime}$ and $k_{i+1}^{\prime}$ into two regions, we get error dynamics of the form

$$
\begin{aligned}
\bar{\zeta}_{k+1} & =\left(A_{m}-K_{m} C_{m}\right) \bar{\zeta}_{k}, k \in\left\{k_{i}^{\prime}, \ldots, k_{i+1}-1\right\} \\
\bar{\zeta}_{k+1} & =\left(A_{m}-K_{m} C_{m}\right) \bar{\zeta}_{k}+\left[\left(A_{m}-A_{l}\right)\right. \\
& \left.-K_{m}\left(C_{m}-C_{l}\right)\right] \bar{x}_{k}, k \in\left\{k_{i+1}, \ldots, k_{i+1}^{\prime}-1\right\}
\end{aligned}
$$

where $\bar{x}=E[x]$. The second term in Eq.(11) arises because a Kalman filter designed for the discrete state $m$ is being used to estimate the dynamics of the discrete state $l$. Combining Eq. (11), we express the error dynamics by $\bar{\zeta}_{k+1}=\left(A_{m}-\right.$ $\left.K_{m} C_{m}\right) \bar{\zeta}_{k}+u_{k}, k \in\left\{k_{i}^{\prime}, \ldots, k_{i+1}^{\prime}-1\right\}$ where

$$
u_{k}=\left\{\begin{array}{c}
0, \text { for } k \in\left\{k_{i}^{\prime}, \ldots, k_{i+1}-1\right\} \\
\left(\left(A_{m}-A_{l}\right)-K_{m}\left(C_{m}-C_{l}\right)\right) \bar{x}_{k}, \\
\quad \text { for } k \in\left\{k_{i+1}, \ldots, k_{i+1}^{\prime}-1\right\}
\end{array}\right.
$$

From this, we get:

$$
\begin{aligned}
\left\|\bar{\zeta}_{k+1}\right\| & \leq\left\|\left(A_{m}-K_{m} C_{m}\right)^{k+1-k_{i}^{\prime}} \bar{\zeta}_{k_{i}^{\prime}}\right\| \\
& +\left\|\sum_{l=0}^{k-k_{i}^{\prime}}\left(A_{m}-K_{m} C_{m}\right)^{k-k_{i}^{\prime}-l} u_{k_{i}^{\prime}+l}\right\|
\end{aligned}
$$

where $k \in\left\{k_{i+1}, \ldots, k_{i+1}^{\prime}-1\right\}$.

Lemma 5 Given a matrix $A \in \mathbb{R}^{n \times n}$ with all distinct eigenvalues,

$$
\left\|A^{t}\right\| \leq k(A) \alpha^{t}(A), \forall t \geq 0
$$

where $\alpha(A)$ is the maximal absolute value of the eigenvalues of $A$, and $k(A)=\|Q\|\left\|Q^{-1}\right\|$, the condition number of $A$ under the inverse, where $Q^{-1} A Q=J$, the Jordan canonical form.

Further simplification of Eq.(13) using Lemma 5 gives us

$$
\begin{aligned}
\left\|\bar{\zeta}_{k+1}\right\| & \leq k\left(A_{m}-C_{m}\right)\left[\alpha\left(A_{m}-K_{m} C_{m}\right)\right]^{k+1-k_{i}^{\prime}}\left\|\bar{\zeta}_{k_{i}^{\prime}}\right\| \\
& +k\left(A_{m}-C_{m}\right) \max \left\|u_{k}\right\|\left(k-k_{i+1}\right)
\end{aligned}
$$

where $k \in\left\{k_{i+1}, \ldots, k_{i+1}^{\prime}\right\}$. Since $k_{i+1}^{\prime}-k_{i+1} \leq \Delta$, if

$$
\left\|u_{k}\right\|_{\infty} \leq U=\max \left\|\left(A_{m}-A_{l}\right)-K_{m}\left(C_{m}-C_{l}\right)\right\|_{1} X
$$

such that $X \geq\|x\|_{\infty}, X>0$, we can write

$$
\begin{aligned}
\left\|\bar{\zeta}_{k+1}\right\| \leq & k\left(A_{m}-C_{m}\right)\left[\alpha\left(A_{m}-K_{m} C_{m}\right)\right]^{k+1-k_{i}^{\prime}}\left\|\bar{\zeta}_{k_{i}^{\prime}}\right\| \\
& +\sqrt{n} U \Delta k\left(A_{m}-C_{m}\right)
\end{aligned}
$$

Lemma 6 Consider a hybrid system with a single discrete state, in which the discrete-time evolution of the continuous state variable is given by $x_{k+1}=\eta x_{k},|\eta|<1$. Suppose the state $x$ is subject to resets $x\left(t_{s}\right)=a \eta x\left(t_{s}-1\right)+b$, occurring at switching times $\left\{t_{s}\right\}$, with $a \geq 1$ and $b \geq 0$. Then the evolution of $x$ can be described by

$$
\begin{aligned}
x_{k} & =\eta^{k-t_{s-1}} x_{t_{s-1}}, k \in\left\{t_{s-1}, \ldots, t_{s}-1\right\} \\
x_{t_{s}} & =a \eta^{t_{s}-t_{s-1}} x_{t_{s-1}}+b
\end{aligned}
$$

Let us also assume there exists a lower bound $\beta$ on the time between resets, i.e., $t_{s}-t_{s-1} \geq \beta \geq 1$, for all $s>1$. Then, if $x_{t_{0}}>0$ and $\mu=\eta^{\left(\frac{\log _{\eta} a}{\beta}+1\right)}$ such that $|\mu|<1$, then $x(k)$ converges exponentially to the set $\left[0, \frac{b}{1-\eta^{\beta}}\right]$ with a rate of convergence greater than or equal to $\mu$.

Using Eqs. (10), (16) and (17) with Lemma 5 and Lemma 6 , we arrive at the following theorem:

Theorem 2 Consider a stochastic linear hybrid system of the form in Eq.(1), a steady-state error bound $M_{0}$ and rate of convergence $\mu,|\mu|<1,\left|\alpha\left(A_{m}-K_{m} C_{m}\right)\right| \leq|\mu|$ for all $m=1 \ldots N$, where $\alpha(A)$ is the maximal absolute value of the eigenvalues of $A$. Let $k(A)=\|Q\|\left\|Q^{-1}\right\|$, the condition number of $A$ under the inverse, where $Q^{-1} A Q=J$, the Jordan canonical form. Then if the following five conditions are satisfied:

1. The system is observable under the definition in Section 2

2. $\left\{A_{m}, C_{m}\right\}$ couples are observable, $\left\{A_{m}, Q_{m}^{1 / 2}\right\}$ couples are controllable, and $\left(A_{m}-K_{m} C_{m}\right)$ is stable with all distinct eigenvalues, for all $m=1 \ldots N$.

3. There exists $X>0,\left\|x_{k}\right\|_{\infty} \leq X, k=1,2, \ldots$ such that $\left\|u_{k}\right\|_{\infty} \leq U=\max \left\|\left(A_{m}-A_{l}\right)-K_{m}\left(C_{m}-C_{l}\right)\right\|_{1} X$

4. The discrete decision time, $\Delta$ satisfies the relation

$$
\Delta \leq \frac{M_{0}}{\sqrt{n} U \max \left[k\left(A_{m}-K_{m} C_{m}\right)\right]}
$$

5. The time between switching events, $\beta$ satisfies the conditions

$$
\begin{aligned}
& \beta>\beta_{\text {min }}+\Delta \text {, where } \\
& \beta_{\text {min }}>\max \left[\frac{1}{|\log \mu|} \log \left|\left(1-\frac{\sqrt{n} U \Delta k\left(A_{m}-K_{m} C_{m}\right)}{M_{0}}\right)\right|\right. \\
& \left., \max \frac{\log \left[k\left(A_{m}-K_{m} C_{m}\right)\right]}{\left[\log \left[\alpha\left(A_{m}-K_{m} C_{m}\right)\right]\right]}\right]
\end{aligned}
$$

we can design a hybrid estimator that converges to within the steady-state bound $M_{0}$ with a rate of convergence greater than or equal to $\mu$.

Corollary 1 If Conditions (1)-(4) of Theorem 3 are satisfied, then, given a steady-state error bound $M_{0}$ and a rate of convergence $\mu$, we can design an estimator that converges exponentially to $M_{0}$ with a rate of at least $\mu$ if the time between switching events is at least $\beta=\beta_{\min }+\Delta$, where

$$
\begin{aligned}
\beta_{\text {min }}=\max & {\left[\frac{1}{|\log \mu|} \log \left|\left(1-\frac{\sqrt{n} U \Delta k\left(A_{m}-K_{m} C_{m}\right)}{M_{0}}\right)\right|,\right.} \\
& \left.\max \frac{\log \left[k\left(A_{m}-K_{m} C_{m}\right)\right]}{\left.\mid \log \left[\alpha\left(A_{m}-K_{m} C_{m}\right)\right]\right]}\right]
\end{aligned}
$$

\section{Example: Aircraft Trajectory}

We apply the above design criteria to the design of an estimator for the switched, linearized trajectory of an aircraft. We consider two discrete states, both coordinated turns, but with different angular velocities, one with a turn rate of $2^{\circ}$ per second, and the other with a turn rate of $5^{\circ}$ per second, which represent aircraft trajectories composed of slow turns and sharp 

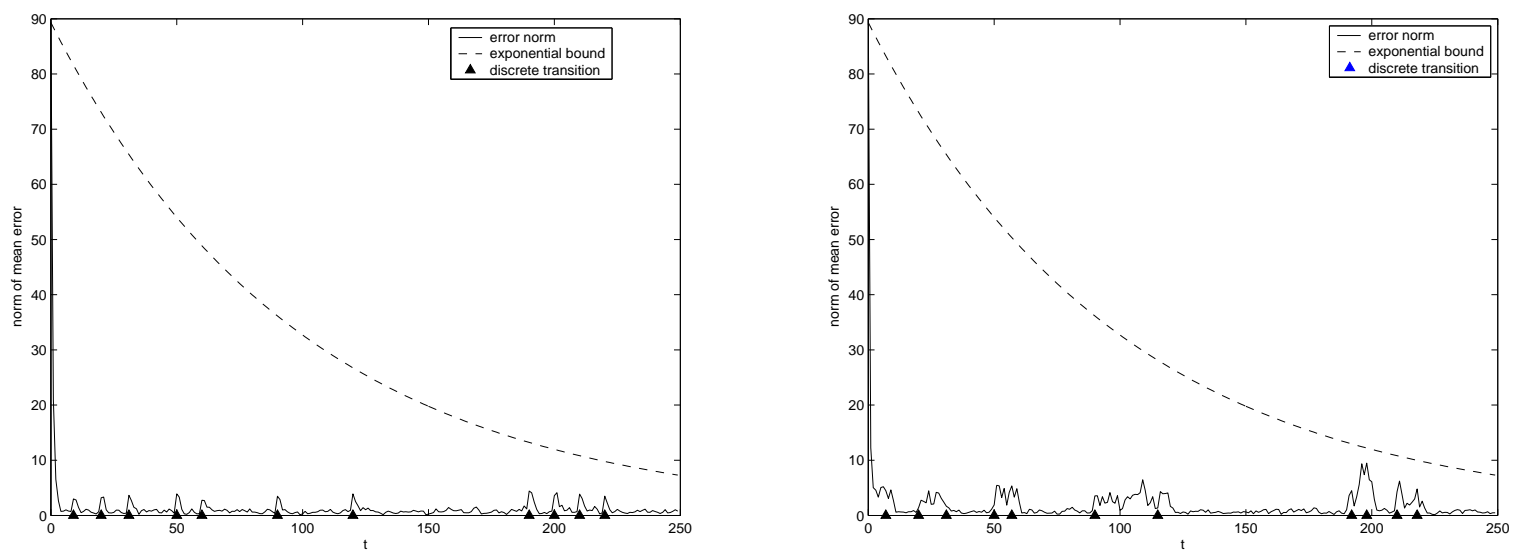

Figure 2: (left) Exponential convergence of error. (right) Convergence of error when modes have same dynamics but different noise characteristics. The triangles denote discrete transition times $\left(\mu=0.99, M_{0}=0\right.$, and $\left.T=2 s e c\right)$.

turns. For brevity, we only include this two discrete state example in this paper but we have successfully designed hybrid estimators for aircraft trajectory tracking and conflict detection and resolution problems with multiple discrete states, such as constant velocity straight flight modes with different noise characteristics and coordinated turn modes with various angular velocities. The dynamics of a coordinated turn is given by

$$
\begin{aligned}
x_{k}= & {\left[\begin{array}{cccc}
1 & \frac{\sin \omega T}{\omega} & 0 & -\frac{1-\cos \omega T}{\omega} \\
0 & \cos \omega T & 0 & -\sin \omega T \\
0 & \frac{1-\cos \omega T}{\omega} & 1 & \frac{\sin \omega T}{\omega} \\
1 & \sin \omega T & 0 & \cos \omega T
\end{array}\right] x_{k-1} } \\
& +\left[\begin{array}{cccc}
\frac{T^{2}}{2} & T & 0 & 0 \\
0 & 0 & \frac{T^{2}}{2} & T
\end{array}\right]^{T} u_{k-1}+w_{k} \\
y_{k}= & {\left[\begin{array}{llll}
1 & 0 & 0 & 0 \\
0 & 0 & 1 & 0
\end{array}\right] x_{k}+v_{k} }
\end{aligned}
$$

where $x=\left[\begin{array}{llll}x_{1} & \dot{x}_{1} & x_{2} & \dot{x}_{2}\end{array}\right]$ where $x_{1}$ and $x_{2}$ are the position coordinates, $u=\left[\begin{array}{ll}u_{1} & u_{2}\end{array}\right]^{T}$ where $u_{1}$ and $u_{2}$ are the velocity components, $\omega$ is the turn rate, $T$ is the sampling interval, $w$ is the process noise, and $v$ is the sensor noise. We choose an operating velocity of 150 knots. We find that for an instantaneous discrete decision time, the time between discrete transitions should be at least 8 seconds to guarantee exponential convergence with a rate of 0.99 . The comparison of the bounds is shown in Figure 2-(left). We also note that by Lemma 5 the norm of the mean error does not have to be monotonic, but if the conditions explained above are satisfied, it will be bounded by an exponential of rate $\mu$. This is also seen in the example. As explained in the Section 2, Lemma 2, identical dynamics with different noise characteristics in each discrete state might still make the system observable in the stochastic hybrid context. We demonstrate this by designing an exponentially convergent hybrid estimator for a switched aircraft trajectory - the two discrete states correspond to $2^{\circ}$ per second turns with different process noise covariances. This is shown in Figure 2-(right).

\section{Conclusions}

In this paper, we have extended the definition of observability to include stochastic linear hybrid systems, and have used prior knowledge of system noise characteristics to im- prove the observability conditions for a discrete-time stochastic linear hybrid system. We have also found bounds on the time between discrete transitions to guarantee the exponential convergence of hybrid estimators for such systems. An interesting direction for future work would be the extension of these results to hybrid systems with continuous state resets.

\section{References}

[1] Y. Bar-Shalom and X.R. Li. Estimation and Tracking: Principles, Techniques, and Software. Artech House, Boston, 1993.

[2] T. Kailath. Linear Systems. Prentice Hall, 1980.

[3] P.J. Ramadge. Observability of discrete event-systems. In Proceedings of the $25^{\text {th }}$ IEEE Conference on Decision and Control, pages 1108-1112, Athens, Greece, 1986.

[4] C.M. Özveren and A.S. Willsky. Observability of discrete event dynamic systems. IEEE Transactions on Automatic Control, 35:797-806, 1990.

[5] A. Alessandri and P. Coletta. Design of Luenberger observers for a class of hybrid linear systems. In M. D. DiBenedetto and A. SangiovanniVincentelli, editors, Hybrid Systems: Computation and Control. LNCS, volume 2034, pages 7-18. Springer-Verlag, 2001.

[6] A. Balluchi, L. Benvenuti, M.D. Di Benedetto, and A.L. SangiovanniVincentelli. Design of observers for hybrid systems. In C. Tomlin and M.R. Greenstreet, editors, Hybrid Systems: Computation and Control. LNCS, volume 2289, pages 76-89. Springer-Verlag, 2002.

[7] A. Bemporad, G. Ferrari, and M. Morari. Observability and controllability of piecewise affine and hybrid systems. IEEE Transactions on Automatic Control, 45(10):1864-1876, 2000.

[8] R. Vidal, A. Chiuso, S. Soatto, and S. Sastry. Observability of linear hybrid systems. In Hybrid Systems: Computation and Control. LNCS. Springer-Verlag, 2003. submitted.

[9] Y. Baram and T. Kailath. Estimability and regulability of linear systems. IEEE Transactions on Automatic Control, 33(12):1116-1121, 1988.

[10] L.R. Rabiner and B.H. Juang. An introduction to Hidden Markov Models. IEEE Transactions on Acoustics, Speech, and Signal Processing, 3(1):4-16, 1986.

[11] E.F. Cost and J.B.R. do Val. On the detectability and observability of discrete-time markov jump linear systems. In Proceedings of the $39^{\text {th }}$ IEEE Conference on Decision and Control, Sydney, Australia, December 2000.

[12] R. Vidal, A. Chiuso, and S. Soatto. Observability and identifiability of jump linear systems. In Proceedings of the $41^{t h}$ IEEE Conference on Decision and Control, Las Vegas,NV, December 2002.

[13] T. Kailath, A.H. Sayed, and B. Hassibi. Linear Estimation. Prentice Hall, New Jersey, 2000. 